\title{
Heroin Abuse Is Characterized by Discrete Mesolimbic Dopamine and Opioid Abnormalities and Exaggerated Nuclear Receptor-Related 1 Transcriptional Decline with Age
}

\author{
Monika Cs. Horvath, ${ }^{1}$ Gabor G. Kovacs, ${ }^{2}$ Viktor Kovari, ${ }^{2}$ Katalin Majtenyi, ${ }^{2}$ Yasmin L. Hurd, ${ }^{3 *}$ and Eva Keller ${ }^{1 *}$ \\ ${ }^{1}$ Department of Forensic Medicine, Semmelweis University, H-1085, Budapest, Hungary, ${ }^{2}$ National Institute of Psychiatry and Neurology, H-1021, \\ Budapest, Hungary, and ${ }^{3}$ Department of Psychiatry, Mount Sinai School of Medicine, New York, New York 10029
}

\begin{abstract}
Dysfunction of mesocorticolimbic dopaminergic neurons is considered a common feature of all drugs of abuse, yet few investigations have evaluated the dopamine (DA) system in nonstimulant human abusers. We examined mRNA expression levels of DA transporter (DAT), tyrosine hydroxylase (TH), dopamine $\mathrm{D}_{2}$ receptor, $\alpha$-synuclein, and nuclear receptor-related 1 (Nurr1) in discrete mesocorticolimbic and nigrostriatal subpopulations of heroin users and control subjects. The chronic use of heroin was significantly associated with decreased DAT mRNA expression localized to the paranigral nucleus (PN) and the mesolimbic division of the ventral tegmental area (VTA) with no alterations in nigrostriatal populations. Consistently, the density of DAT immunoreactivity was significantly reduced in the nucleus accumbens but not in dorsal striatum, mesolimbic and nigrostriatal efferent targets, respectively. Significant alteration of the mRNA expression of Nurrl, a transcription factor that regulates DAT expression, was also confined to the PN. Moreover, the results revealed an exaggerated reduction of Nurrl expression with age in heroin users $(r=-0.8268, p<0.001$ vs controls, $r=-0.6204, p=$ 0.0746). TH and $\alpha$-synuclein mRNA levels were, in contrast, elevated in the VTA PN in heroin users with no change of the $\mathrm{D}_{2}$ receptor. Evaluating midbrain $\mu$-and $\kappa$-opioid receptors, relevant for the action of heroin and regulation of DA neurons, revealed dysregulation of G-protein coupling selective to the VTA PN. Altogether the current findings provide direct neurobiological evidence that midbrain reward circuits have the most prominent DA and opioid impairments in human heroin abusers and that abnormal Nurrl transcription with opiate use may exacerbate limbic dysfunction with age.
\end{abstract}

Key words: dopamine transporter; $\alpha$-synuclein; human; $\mu$-opioid receptor; substantia nigra; periaqueductal gray

\section{Introduction}

In addition to the direct stimulation of $\mu$-opioid receptors (MORs) (and $\delta$ ) in the ventral striatum (nucleus accumbens), indirect regulation of mesocorticolimbic dopamine (DA) neurotransmission has also been strongly implicated in the pathophysiology of opioid addiction. Stimulation of MORs localized on inhibitory GABAergic neurons in the midbrain ventral tegmental area (VTA) leads to enhanced DAergic activity (Johnson and North, 1992) and subsequent elevation of DA levels in projection terminals such as the nucleus accumbens (Spanagel et al., 1990; Pontieri et al., 1995). The VTA is heterogeneous with distinct anatomical and functional organization. In addition to rewardrelated mesolimbic DA neurons that innervate the nucleus accumbens, a mesocortical subpopulation projects to the prefron-

\footnotetext{
Received May 25, 2007; revised Sept. 20, 2007; accepted 0ct. 9, 2007.

This work was supported by Hungarian Scientific Health Council GrantETT 236/2003, National Institutes of Health Grant NIDA DA15446, Hungarian Scientific Research Fund Grant 0TKA T32227, European Union Sixth Framework Programme, BNEll No. LSHM-CT-2004-503039 (G.G.K.). M.Cs.H. was supported by the Swedish Institute and the Hungarian Scholarship Board. We are grateful to Mariann Lenkeiné and Alexandra Tylec for technical assistance.

*Y.L.H. and E.K. contributed equally to this work.

Correspondence should be addressed to Yasmin L. Hurd, Department of Psychiatry, Mount Sinai School of Medicine, One Gustave L. Levy Place, Box 1215, New York, NY 10029. E-mail: Yasmin.Hurd@mssm.edu. DOI:10.1523/JNEUROSCI.2398-07.2007

Copyright $\odot 2007$ Society for Neuroscience $\quad$ 0270-6474/07/2713371-05\$15.00/0
}

tal cortex relevant for cognitive function and emotional regulation (Swanson, 1982; Haber and Fudge, 1997). DA neurons are primarily characterized by expression of the DA transporter (DAT), a transmembrane protein that mediates the reuptake of DA, thereby regulating the actions of synaptic (and extrasynaptic) DA in the brain.

In addition to DAT, tyrosine hydroxylase $(\mathrm{TH})$ (rate-limiting enzyme for DA synthesis), and $\mathrm{DA} \mathrm{D}_{2}$ receptor (negative autoregulator of DA cell firing), $\alpha$-synuclein and nuclear receptorrelated 1 (Nurr1) have been recently identified as important regulators of DA function. $\alpha$-Synuclein forms complexes with DAT (Wersinger et al., 2003), regulates the amount of DAT in the plasma membrane (Wersinger et al., 2003), and modulates DA biosynthesis by reducing the activity of TH (Perez et al., 2002). Dysfunction of $\alpha$-synuclein, primarily localized to substantia nigra DA neurons, has been highly implicated in Parkinson's disease, but only few studies have investigated its potential involvement in drug addiction. Of these, an overexpression of $\alpha$-synuclein was observed in DA neurons of cocaine users (Mash et al., 2003). Nurr1 is a member of the nuclear receptor superfamily of transcription factors critical for development and maintenance of DA cells (Perlmann and Wallén-Mackenzie, 2004). Abundant Nurr1 has been demonstrated in the adult midbrain (Bannon et al., 2002) and suggested to be necessary for the regu- 
lation of DAT (Smits et al., 2003) and TH (Kim et al., 2003). Decreased Nurr1 transcription was detected in human cocaine abusers (Bannon et al., 2002), but no studies to date have investigated Nurr1 in relation to opiates.

Given the important implications of DA neurotransmission in addiction disorders and the relative paucity of data regarding the DA system in relation to human opiate addiction, we examined phenotypic DA markers in mesolimbic and mesocortical VTA subpopulations in heroin abusers as well as nigrostriatal neurons, generally linked to neurodegenerative disorders and habit learning underlying compulsive drug use (Ito et al., 2002; Faure et al., 2005). MOR and $\kappa$-opioid receptor (KOR) function was also evaluated because of their role in mediating the actions of heroin and regulating midbrain DA neurons (Bals-Kubik et al., 1993; Ford et al., 2007).

\section{Materials and Methods}

Human brain specimens. Brain specimens from Caucasian subjects were obtained at autopsy from the Forensic Medicine Department of Semmelweis University (Budapest, Hungary) and the Forensic Medicine Department of Karolinska Institutet (Stockholm, Sweden) under approved local ethical guidelines. All cases had a postmortem interval of $<24 \mathrm{~h}$ and were evaluated for common drugs of abuse (including alcohol) and therapeutic drugs. Brain samples were processed for two analysis conditions: subset I $(n=32)$, fresh-frozen, for molecular mRNA determination; and subset II $(n=27)$, formalin-fixed, for immunohistochemical evaluation (supplemental methods and Table 2, available at www.jneurosci.org as supplemental material). Subjects were classified into two main case groups according to their cause of death: control and heroin overdose. The heroin group represented a unique drug abuse population with predominant heroin use and with no history of methadone or buprenorphine clinical treatment; information was obtained from family and/or available medical documentation. They were also negative for human immunodeficiency virus (HIV) infection. The control group had negative toxicology for opiates or other drugs of abuse, except alcohol in very few cases in which ethanol concentrations were similar to the limited alcohol-positive subjects identified in the heroin group.

In situ hybridization histochemistry and $\left[{ }^{35} S\right] G T P \gamma S$ autoradiography. Duplicate brain sections (20- $\mu \mathrm{m}$-thick) per subject from subset I were processed through in situ hybridization histochemistry (Fagergren et al., 2003) using ${ }^{35}$ S-labeled RNA probes to determine the relative expression of mRNA levels of DAT, TH, DA $D_{2}$, Nurr1, $\alpha$-synuclein, and calbindin. Detailed information of the brain preparations, probes, and in situ hybridization procedures are provided in the supplemental methods, available at www.jneurosci.org as supplemental material. $\left[{ }^{35} \mathrm{~S}\right] \mathrm{GTP} \gamma \mathrm{S}$ autoradiography was performed to determine basal and opioid agonistinduced GTP $\gamma S$ binding (supplemental methods, available at www.jneurosci.org as supplemental material).

Immunohistochemistry. Immunohistochemistry was performed on subset II samples to measure DAT protein levels [rabbit polyclonal (1: 100; Millipore, Billerica, MA) detects the C terminal of the DAT]; details are in supplemental methods, available at www.jneurosci.org as supplemental material.

Quantification. Full details are provided in supplemental methods, available at www.jneurosci.org as supplemental material. In brief, five DA cell groups were identified in the human midbrain: limbic-related "dorsal tier" nuclei included the VTA [consisting of the paranigral nucleus (PN) and parabrachial pigmented (PBP) nucleus] and the substantia nigra dorsal part (SNd), and motor-related "ventral tier" nuclei included the substantia nigra ventral part ( $\mathrm{SNv}$ ) and substantia nigra lateral part (SNl) (supplemental Fig. 1, available at www.jneurosci.org as supplemental material) (Haber and Fudge, 1997). The subpopulations were distinguished by the coregister with the distribution pattern of calbindin mRNA expression, which was present in dorsal tier but not in ventral tier neuronal populations (supplemental Fig. $1 A$, available at www. jneurosci.org as supplemental material). Measurements from duplicates and right and left sides were averaged to one value/subject for each sub-
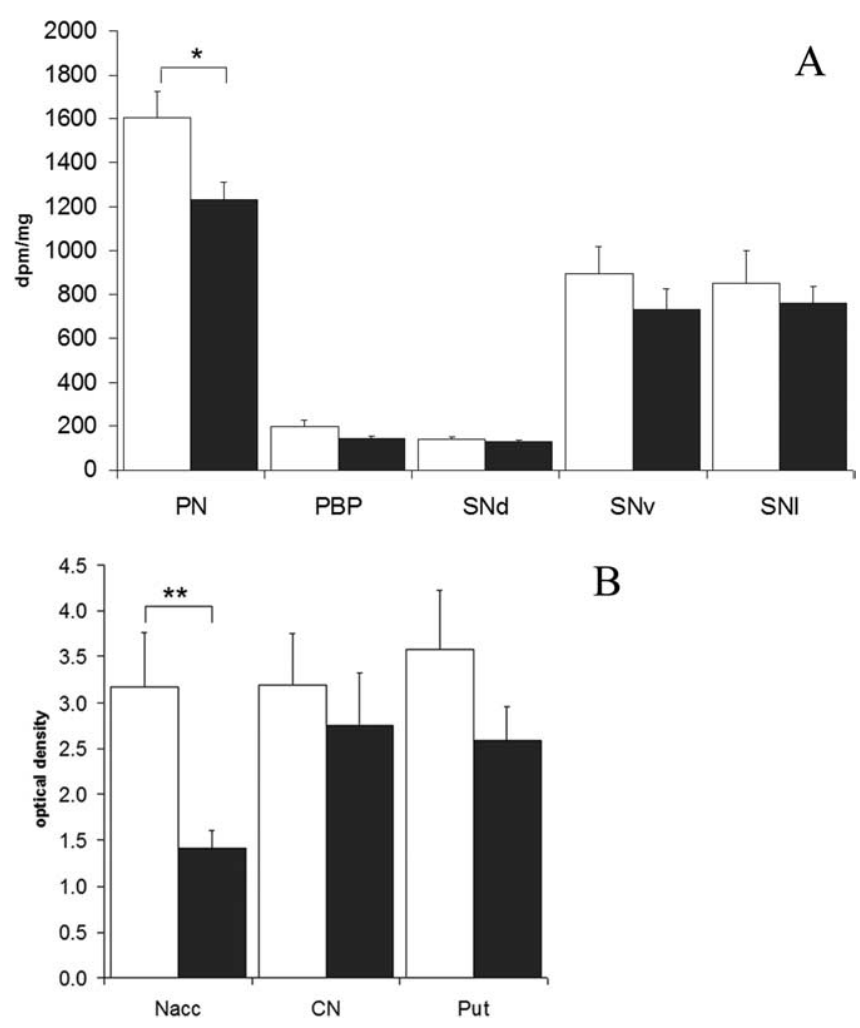

B

Figure 1. mRNA expression levels $(\boldsymbol{A})$ and protein density levels $(\boldsymbol{B})$ of DAT in subregions of the midbrain and striatum in control (white) and heroin (black) groups. ${ }^{*} p<0.05 ;{ }^{* *} p<0.01$. $A, P N$ covaried for freezing time. Nacc, Nucleus accumbens; $C \mathrm{~N}$, caudate nucleus; Put, putamen. Error bars represent SEM.

region. Based on the known radioactivity in coexposed standards, optical density values were converted to disintegrations per minute per milligram (mRNA expression) and microcuries per milligram (receptor binding). Immunoreactivity for DAT was quantified in the caudate nucleus, putamen, and nucleus accumbens (core and shell subdivisions). Univariate analyses and general linear stepwise regression analyses [using JMP version 3.1 (SAS Institute, Cary, NC) and Statistica 6.1 (StatSoft, Tulsa, OK) software packages] were used to determine group differences in DAand opioid-related markers in subpopulations of the brainstem as well as in density values of DAT in the striatum. Independent variables (age, postmortem interval, sex, brain $\mathrm{pH}$, freezing time, ethanol toxicity) were included in the model if the univariate analysis showed the variable to be significant $(p<0.05)$.

\section{Results}

\section{Mesolimbic DAT impairment in heroin abusers}

Heroin subjects had a significant reduction of DAT mRNA expression in the PN $\left(F_{(2,22)}=7.4836, p=0.0263\right.$, covaried for freezing time). A similar direction of change, with strong trend effect, was also observed in the other dorsal tier subregions, namely the PBP $(p=0.0515)$ and $\mathrm{SNd}(p=0.0768)$. No significant group effect was detected in the ventral tier subnuclei (Fig. $1 A)$. There was no correlation to morphine toxicology. Given the strong mesolimbic specificity of DAT mRNA in heroin abusers, DAT immunoreactivity was examined to assess the relevance to protein levels. The nucleus accumbens and dorsal striatum, the major efferent targets of the mesolimbic and nigrostriatal pathways, respectively, were studied in the same striatal section. DAT immunoreactivity showed a heterogeneous distribution pattern characteristic of the human striatum. In heroin subjects, the density of DAT immunoreactivity was significantly decreased by $55 \%$ in the nucleus accumbens compared with controls $(p=0.002)$ 


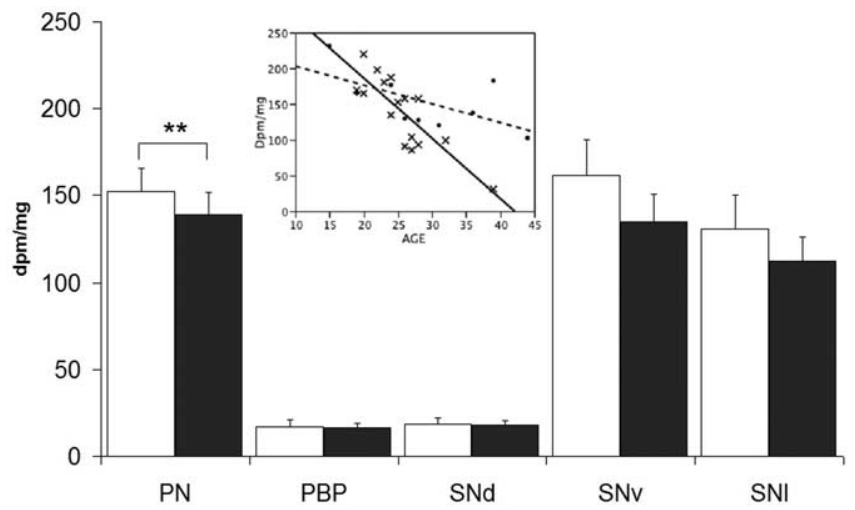

Figure 2. mRNA expression levels of Nurr1 (PN covaried for age and ethanol toxicology; age $\times$ group interaction) in control (white) versus heroin (black) groups. Inset, Correlation between Nurr1 disintegrations per minute per milligram values and age in the PN of control (dashed line, filled circles; $r=-0.08268 ; p<0.001$ ) and heroin (solid line, $X ; r=-0.6204$; $p=0.0746$ ) subjects. ${ }^{*} p<0.05 ;{ }^{* *} p<0.01$. Error bars represent SEM.

(Fig. $1 B)$. No significant changes were observed in the caudate nucleus $(p=0.53)$ and putamen $(p=0.16)$. Examination of the discrete subregions of the nucleus accumbens showed significant reduction in both the core $(42 \%)$ and shell $(39 \%)(p<0.01)$ of the heroin users. No demographic variable was found to influence the DAT density in any of the striatal subregions examined ( $p>0.1$ in all comparisons). However, there was a nonsignificant trend for an inverse correlation between DAT density in the nucleus accumbens and blood codeine levels in heroin subjects $(n=13 ; r=-0.52 ; p=0.06)$.

\section{Midbrain expression levels of Nurr1, $\alpha$-synuclein, TH, and} $\mathrm{D}_{2}$ transcripts

Alterations of the Nurr1 gene expression were confined to the PN in which the mRNA levels were significantly reduced $\left(F_{(4,24)}=\right.$ $13.711 ; p=0.0089$ ) in the heroin group (Fig. 2). Moreover, age $(p<0.0001)$ markedly influenced the Nurr1 mRNA expression levels and there was a strong significant group $\times$ age interaction $(p=0.0012)$ such that Nurr1 mRNA levels were reduced to a greater extent in the PN of heroin subjects $(r=-0.8268$; $p<$ 0.001 ) with increasing age than that observed for the control group $(r=-0.6204 ; p=0.0746)$ (Fig. 2 , inset). The Nurr1 decline with age was still evident in heroin subjects when examining only the youngest individuals ( $<30$ years old; $n=14 ; r=$ $-0.7099 ; p=0.0044)$. No significant differences were detected for the Nurr1 mRNA expression between heroin and control subjects in the other midbrain DAergic subpopulations (Fig. 2).

In contrast to the reduction observed for the other DA-related markers, expression of both TH and $\alpha$-synuclein mRNAs were elevated in discrete midbrain nuclei. TH mRNA expression was significantly increased in the $\mathrm{PN}\left(F_{(2,25)}=5.1965 ; p=0.0494\right)$, $\operatorname{SNd}\left(F_{(1,28)}=4.5344 ; p=0.0425\right)$, and SNl $\left(F_{(2,20)}=7.0989 ; p=\right.$ 0.0035 ) of the heroin users (supplemental Table 2, available at www.jneurosci.org as supplemental material). Significant elevation of $\alpha$-synuclein mRNA expression levels was observed in the $\mathrm{PN}\left(F_{(3,22)}=6.3153 ; p=0.0035\right)$ and $\mathrm{SNv}\left(F_{(2,23)}=6.5692 ; p=\right.$ 0.0499 ) (supplemental Table 2, available at www.jneurosci.org as supplemental material).

Expression levels of the $\mathrm{DA}_{2}$ receptor mRNA did not differ between the heroin and control groups in any of the brainstem subregions studied (supplemental Table 2, available at www. jneurosci.org as supplemental material).
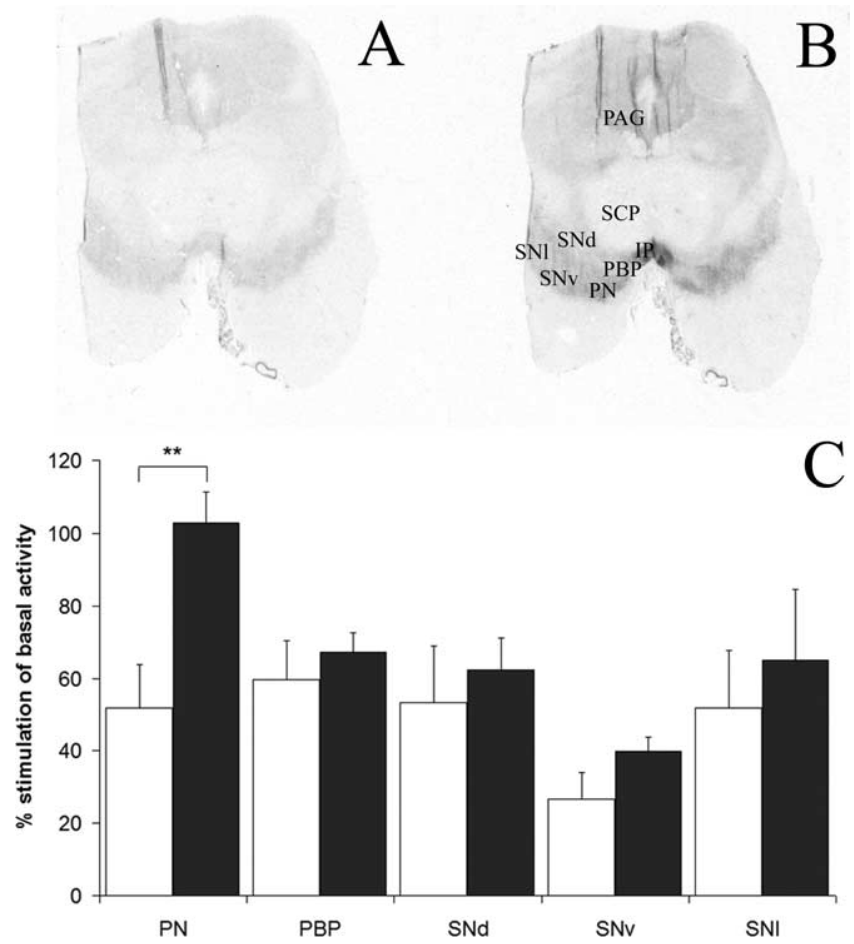

Figure 3. Representative film autoradiograms of the human midbrain showing $\left[{ }^{35} S\right] G T P \gamma S$ coupling under basal $(\boldsymbol{A})$ and DAMGO-stimulated $(\boldsymbol{B})$ conditions. $\boldsymbol{C}$, The bar graph shows percentage of stimulation of $\left[{ }^{35} \mathrm{~S}\right] \mathrm{GTP} \gamma \mathrm{S}$ binding by DAMGO in specific neuronal populations of control (white) versus heroin (black) groups. ${ }^{*} p<0.05$; ${ }^{* *} p<0.01$; ${ }^{* * *} p<0.001$. SCP, Superior cerebellar peduncle; $C P$, cerebral peduncle; $I P$, interpeduncular nucleus. Error bars represent SEM.

None of the expression levels of the DA-related mRNAs examined were significantly correlated to morphine toxicology.

\section{Dysfunction of midbrain mesolimbic MOR and KOR functional coupling in heroin users}

To assess opioid receptor function in the midbrain relevant to the regulation of mesocorticolimbic and nigrostriatal DA tone, G-protein coupling was evaluated by opioid agonist-induced GTP $\gamma \mathrm{S}$ stimulation. In addition to the DA-related cell populations and consistent with animal studies, opioid receptor binding was also visible in the periaqueductal gray (PAG) (well documented to be involved in nociception) and interpeduncular nucleus (prominent output of the habenula implicated in drug reward, separate from the mesolimbic pathway) (Glick et al., 2006) (supplemental Table 2, available at www.jneurosci.org as supplemental material). Basal levels of G-protein binding were significantly higher in the PN $\left(F_{(2,26)}=13.266 ; p=0.0062\right)$ and $\mathrm{PAG}$ $\left(F_{(2,19)}=15.727 ; p=0.047\right)$ of heroin users with a trend effect in the PBP $(p=0.067)$ (supplemental Table 2, available at www.jneurosci.org as supplemental material). D-Ala ${ }^{2}-\mathrm{N}-\mathrm{Me}-\mathrm{Phe}^{4}$ Glycol ${ }^{5}$-enkephalin (DAMGO)-stimulated $\left[{ }^{35} \mathrm{~S}\right] \mathrm{GTP} \gamma \mathrm{S}$ binding revealed a strong significant enhancement of MOR coupling in the PN $\left(F_{(1,30)}=11.9031 ; p=0.0017\right)$ (Fig. 3$)$ and PAG $\left(F_{(1,19)}=\right.$ 29.4512; $p<0.0001$ ) (supplemental Table 2, available at www. jneurosci.org as supplemental material) of heroin users compared with controls. No other midbrain subregion examined was significantly altered. Activation of $\left[{ }^{35} \mathrm{~S}\right] \mathrm{GTP} \gamma \mathrm{S}$-binding by U69593 also showed a significant elevation of KOR coupling in the PN $\left(F_{(1,27)}=8.9853 ; p=0.0059\right)$ and $\operatorname{PAG}\left(F_{(3,19)}=5.277\right.$; $p=0.03789$ ) of heroin users (supplemental Table 2, available at 
www.jneurosci.org as supplemental material). Morphine or codeine concentrations in blood or urine of heroin users were not significantly correlated to either DAMGO- or U69593stimulated $\left[{ }^{35} \mathrm{~S}\right] \mathrm{GTP} \gamma \mathrm{S}$ binding levels.

\section{Discussion}

This study provides direct neurobiological evidence that discrete subpopulations of VTA neurons are impaired in the human midbrain in association with chronic heroin use relevant to reward function. The distribution of mesocorticolimbic DA cells is segregated such that $\mathrm{PN}$ neurons predominantly comprise the mesolimbic pathway, whereas the PBP and SNd constitute the mesocortical circuit (Simon et al., 1979; Pickel et al., 2002). Of the neuronal populations examined, the VTA PN subdivision was consistently altered for most of the DA markers examined: DAT, Nurr1, TH, and $\alpha$-synuclein. Although there was a lack of significant DA-related alterations in the PBP, there was a strong trend for reduced DAT mRNA in this subregion, and an upregulation of the TH mRNA expression was apparent in SNd neurons. Thus, disturbance of mesocortical neurons may occur relevant to prefrontal cortical regulation. The fact, however, that no other DArelated marker was altered in the PBP and SNd in contrast to the $\mathrm{PN}$ emphasizes the apparent greater limbic sensitivity in relation to heroin abuse.

DAT has been extensively implicated in drug addiction as a result of its important role in regulating synaptic DA tone and as the primary pharmacological target for psychostimulant drugs. The parallel decrease of DAT mRNA expression in the PN and protein levels in the projection terminals of the nucleus accumbens highlights the significant role of mesolimbic DAT even in opiate abuse. Although there was a tendency for reduced DAT levels in nonlimbic areas, there were no significant alterations in the dorsal striatum for the protein or for the corresponding mRNA expression in the $\mathrm{SNv}$ and $\mathrm{SNl}$ cell body regions. Despite the strong link between impaired DA function and drug abuse, only one human postmortem study evaluated the DA system in heroin users (Kish et al., 2001), and decreased DAT binding affinity was reported in the nucleus accumbens consistent with the mesolimbic DAT disturbance detected in our study.

The downregulation currently observed in heroin abusers, both on the level of DAT gene expression and protein in the mesolimbic circuit, was complemented by disturbance of other genes related to DAT. Nurr1 is an important regulator of DAT (Sacchetti et al., 2001), and downregulation of Nurr1 mRNA levels in the heroin users was coincident with diminished DAT transcription in the PN. Our results also revealed a strong agerelated decrease of Nurr1 expression, a finding consistent with previous human and experimental studies (Chu et al., 2002; Jiang et al., 2005). Interestingly, the use of heroin markedly accelerated the normal decrease of Nurr1 expression with increasing age, although the subjects were relatively young. Some reports have suggested that the mesolimbic DA system is more vulnerable to aging than the nigrostriatal pathway (Cruz-Muros et al., 2006), which could account for the exacerbated decrease of mesolimbic Nurr1 expression in subjects in which PN neuronal populations are already dysfunctional because of opiate use. The localized and marked age-related impairment of Nurr1 in the PN of heroin abusers strongly suggest that limbic function could be compromised to a greater extent as these individuals become older.

$\alpha$-Synuclein also regulates DAT (Wersinger et al., 2003), and the increased expression of $\alpha$-synuclein mRNA in our heroin subjects is comparable with other substance abuse human studies showing elevated $\alpha$-synuclein mRNA and protein levels in co- caine abusers (Mash et al., 2003; Qin et al., 2005) and alcoholics (Bonsch et al., 2004, 2005). Increased midbrain $\alpha$-synuclein is characteristic of neurodegeneration of DA pigmented cells (elAgnaf and Irvine, 2002) within the substantia nigra. However, VTA DA neurons appear resistant to $\alpha$-synuclein neurotoxic properties (Maingay et al., 2006), and there is no evidence to our knowledge of brainstem neurodegeneration in heroin abusers. Thus, disturbance of PN $\alpha$-synuclein may be more related to its normal modulation of DAT function than to neurotoxicity.

Vulnerability of the mesolimbic populations was not only apparent for DAT-related markers, but also for MOR, the primary pharmacological target for heroin metabolites. Of the mesocorticolimbic and nigrostriatal populations examined, upregulation of MOR, as well as KOR, G-protein coupling was restricted to the PN. Interestingly, electrophysiological studies have recently revealed significant heterogeneity in the opioid composition and physiological regulation of the different mesocorticolimbic DA subpopulations (Ford et al., 2006; Margolis et al., 2006). It was not currently possible to discern the cellular localization (somatodendritic and axon terminal) of the opioid receptor changes in the human midbrain that would help in interpreting the physiological outcome of the receptor alterations. Generally, MOR and KOR mediate opposite regulation of midbrain DAergic activity (Ford et al., 2007) and behavior (Bals-Kubik et al., 1993). Upregulation of MOR, localized on either GABAergic interneurons and afferents terminals, would be predicted to increase mesolimbic DAergic tone by diminishing the inhibitory GABAergic modulation on DA neurons. KOR is primarily situated on DA neurons, but there is some recent discrepancy as to the midbrain KOR regulation of mesolimbic circuits with report of either inhibition (Ford et al., 2006) or no effect (Margolis et al., 2006) on nucleus accumbens-projecting cells. Although species differences might account for some of these discrepancies, additional studies are clearly needed to explore the apparent complex regulation of KOR. Nevertheless, the fact that the opioid receptor dysfunction was selective to the PN cell population strongly demonstrates discrete vulnerability of mesolimbic circuits that would impact DAergic tone in heroin abusers.

There are limitations of the present study. For example, detailed information regarding the subject's entire medical history that might influence the DAergic markers is unknown. Also, it cannot be excluded that subjects could have used other drugs such as psychostimulants or prescription medications that contributed in part to the DAergic alterations. However, our population of subjects was predominant heroin users with no documented history of stimulant use and lacked methadone or buprenorphine treatment. Moreover, they were also unique in lacking HIV infection, which is known to affect DA neurons (Berger and Arendt, 2000).

In conclusion, chronic opiate use in humans is primarily associated with abnormality of dorsal tier mesocorticolimbic populations rather than a generalized broad dysfunction of brainstem DA cells. The consistent alteration of the VTA PN subdivision observed for opioid receptor coupling and most of the DAergic markers strongly suggest a more profound impairment of the mesolimbic reward circuit in heroin-dependent individuals. The parallel change of DAT mRNA and protein levels in the PN and nucleus accumbens, respectively, emphasizes the continuum of disturbances throughout the mesolimbic circuit. Additional evaluation of Nurr1 and $\alpha$-synuclein is needed to provide insight as to their intracellular regulation of DA neurotransmission, which contributes to limbic dysfunction in opiate addiction, especially in relation to Nurr1 and aging. 


\section{References}

Bals-Kubik R, Ableitner A, Herz A, Shippenberg TS (1993) Neuroanatomical sites mediating the motivational effects of opioids as mapped by the conditioned place preferences paradigm in rats. J Pharmacol Exp Ther 264:489-495.

Bannon MJ, Pruetz B, Manning-Bog AB, Whitty CJ, Michelhaugh SK, Sacchetti P, Granneman JG, Mash DC, Schmidt CJ (2002) Decreased expression of the transcription factor NURR1 in dopamine neurons of cocaine abusers. Proc Natl Acad Sci USA 99:6382-6385.

Berger JR, Arendt G (2000) HIV dementia: the role of the basal ganglia and dopaminergic systems. J Psychopharmacol 14:214-221.

Bonsch D, Reulbach U, Bayerlein K, Hillemacher T, Kornhuber J, Bleich S (2004) Elevated alpha synuclein mRNA levels are associated with craving in patients with alcoholism. Biol Psychiatry 56:984-986.

Bonsch D, Greifenberg V, Bayerlein K, Biermann T, Reulbach U, Hillemacher T, Kornhuber J, Bleich S (2005) Alpha-synuclein protein levels are increased in alcoholic patients and are linked to craving. Alcohol Clin Exp Res 29:763-765.

Chu Y, Kompoliti K, Cochran E, Mufson E, Kordower J (2002) Age-related decreases in Nurrl immunoreactivity in the human substantia nigra. J Comp Neurol 450:203-214.

Cruz-Muros I, Alfonso-Oramas D, Abreu P, Barroso-Chinea P, Rodríguez M, González MC, Hernández TG (2006) Aging of the rat mesostriatal system: differences between the nigrostriatal and the mesolimbic compartments. Exp Neurol 204:147-161.

el-Agnaf O, Irvine G (2002) Aggregation and neurotoxicity of alphasynuclein and related peptides. Biochem Soc Trans 30:559-565.

Fagergren P, Smith HR, Daunais JB, Nader MA, Porrino LJ, Hurd YL (2003) Temporal upregulation of prodynorphin mRNA in the primate striatum after cocaine self-administration. Eur J Neurosci 17:2212-2218.

Faure A, Haberland U, Conde F, El Massioui N (2005) Lesion to the nigrostriatal dopamine system disrupts stimulus-response habit formation. J Neurosci 25:2771-2780.

Ford C, Mark G, Williams J (2006) Properties and opioid inhibition of mesolimbic dopamine neurons vary according to target location. J Neurosci 26:2788-2797.

Ford CP, Beckstead MJ, Williams JT (2007) Kappa opioid inhibition of somatodendritic dopamine inhibitory postsynaptic currents. J Neurophysiol 97:883-891.

Glick S, Ramirez R, Livi J, Maisonneuve I (2006) 18-Methoxycoronaridine acts in the medial habenula and/or interpeduncular nucleus to decrease morphine self-administration in rats. Eur J Pharmacol 537:94-98.

Haber SN, Fudge JL (1997) The primate substantia nigra and VTA: integrative circuitry and function. Crit Rev Neurobiol 11:323-342.

Ito R, Dalley JW, Robbins TW, Everitt BJ (2002) Dopamine release in the dorsal striatum during cocaine-seeking behavior under the control of a drug-associated cue. J Neurosci 22:6247-6253.

Jiang C, Wan X, He Y, Pan T, Jankovic J, Le W (2005) Age-dependent dopaminergic dysfunction in Nurrl knockout mice. Exp Neurol 191:154-162.

Johnson SW, North RA (1992) Opioids excite dopamine neurons by hyperpolarization of local interneurons. J Neurosci 12:483-488.
Kim K, Kim C, Hwang D, Seo H, Chung S, Hong S, Lim J, Anderson T, Isacson O (2003) Orphan nuclear receptor Nurrl directly transactivates the promoter activity of the tyrosine hydroxylase gene in a cell-specific manner. J Neurochem 85:622-634.

Kish S, Kalasinsky K, Derkach P, Schmunk G, Guttman M, Ang L, Adams V, Furukawa Y, Haycock J (2001) Striatal dopaminergic and serotonergic markers in human heroin users. Neuropsychopharmacology 24:561-567.

Maingay M, Romero-Ramos M, Carta M, Kirik D (2006) Ventral tegmental area dopamine neurons are resistant to human mutant alpha-synuclein overexpression. Neurobiol Dis 23:522-532.

Margolis E, Lock H, Chefer V, Shippenberg T, Hjelmstad G, Fields H (2006) Kappa opioids selectively control dopaminergic neurons projecting to the prefrontal cortex. Proc Natl Acad Sci USA 103:2938-2942.

Mash D, Ouyang Q, Pablo J, Basile M, Izenwasser S, Lieberman A, Perrin R (2003) Cocaine abusers have an overexpression of $\alpha$-synuclein in dopamine neurons. J Neurosci 23:2564-2571.

Perez R, Waymire J, Lin E, Liu J, Guo F, Zigmond M (2002) A role for $\alpha$-synuclein in the regulation of dopamine biosynthesis. J Neurosci 22:3090-3099.

Perlmann T, Wallén-Mackenzie A (2004) Nurr1, an orphan nuclear receptor with essential functions in developing dopamine cells. Cell Tissue Res 318:45-52.

Pickel V, Chan J, Nirenberg M (2002) Region-specific targeting of dopamine D2-receptors and somatodendritic vesicular monoamine transporter 2 (VMAT2) within ventral tegmental area subdivisions. Synapse 45:13-24.

Pontieri FE, Tanda G, Di Chiara G (1995) Intravenous cocaine, morphine, and amphetamine preferentially increase extracellular dopamine in the "shell" as compared with the "core" of the rat nucleus accumbens. Proc Natl Acad Sci USA 92:12304-12308.

Qin Y, Ouyang Q, Pablo J, Mash DC (2005) Cocaine abuse elevates alphasynuclein and dopamine transporter levels in the human striatum. Neuroreport 16:1489-1493.

Sacchetti P, Mitchell TR, Granneman JG, Bannon MJ (2001) Nurr1 enhances transcription of the human dopamine transporter gene through a novel mechanism. J Neurochem 76:1565-1572.

Simon H, Le Moal M, Stinus L, Calas A (1979) Anatomical relationships between the ventral mesencephalic tegmentum-a 10 region and the locus coeruleus as demonstrated by anterograde and retrograde tracing techniques. J Neural Transm 44:77-86.

Smits S, Ponnio T, Conneely O, Burbach J, Smidt M (2003) Involvement of Nurr1 in specifying the neurotransmitter identity of ventral midbrain dopaminergic neurons. Eur J Neurosci 18:1731-1738.

Spanagel R, Herz A, Shippenberg TS (1990) The effects of opioid peptides on dopamine release in the nucleus accumbens: an in vivo microdialysis study. J Neurochem 55:1734-1740.

Swanson LW (1982) The projections of the ventral tegmental area and adjacent regions: a combined fluorescent retrograde tracer and immunofluorescence study in the rat. Brain Res Bull 9:321-353.

Wersinger C, Prou D, Vernier P, Sidhu A (2003) Modulation of dopamine transporter function by a-synuclein is altered by impairment of cell adhesion and by induction of oxidative stress. FASEB J 17:2151-2153. 